\title{
Influência da sensibilidade individual de varrões sobre a qualidade espermática após descongelação
}

\section{Influence of boar individual sensibility on spermatic quality after tawing}

\author{
Ricardo Toniolli, ${ }^{*}$ Ana Beatriz Graça Duarte, ${ }^{*}$ Jorge Luiz Ferreira, ${ }^{*}$ Faviano Ricelli da Costa e Moreira*
}

\begin{abstract}
Resumo
Diversos trabalhos têm demonstrado diferenças na qualidade espermática do sêmen descongelado entre diversos reprodutores suínos, demonstrando variações na resistência individual dos espermatozóides destes animais ao processo de congelação. Este trabalho teve por objetivo testar o sêmen proveniente de diferentes reprodutores para se identificar sensibilidades individuais ao processo de congelação. Quatro machos foram coletados, em recipiente de $500 \mathrm{~mL}$ coberto por gaze para separação da parte gelatinosa e protegido por envoltório térmico. Cada amostra continha $112 \times 10^{6} \mathrm{sptz} / \mathrm{mL}$ para as análises in vitro. A técnica de Paquignon foi utilizada para a congelação do sêmen. Após a descongelação, a $37^{\circ} \mathrm{C}$ durante 30 segundos, o conteúdo de cada palheta foi ressuspenso após descongelação no diluente Beltsville Thawing Solution (BTS). Foram avaliadas em microscopia óptica o vigor e a motilidade espermática após descongelação (M1), ressuspensão (M2) e 10 minutos (M3) e 2 horas de incubação (M4) a $37^{\circ} \mathrm{C}$. A morfologia do acrossoma foi analisada em esfregaço de sêmen feito em M3. Foi utilizado o teste de Mann Whitney através do General Linear Models do programa Statistical Analysis System (SAS 6.03, 1988) a 5\% (p<0,05).
\end{abstract}

Palavras-chave: varrões, sêmen, congelação, espermatozoides, sensibilidade.

\begin{abstract}
Several works have been demonstrating differences in spermatic quality of the thawed semen among several swine males. Variations are diagnosed in the individual resistance of the spermatozoids of these animals to the freezing process. This work had for objective to test the semen from the different boars to identify individual sensibilities to the freezing process. Four males were collected, in recipient with $500 \mathrm{~mL}$ covered by filter for separation of the gelatinous part and protected by thermal wrapper. Each sample contained $112 \times 10^{6} \mathrm{sptz} / \mathrm{mL}$ for the in vitro analyses. The Paquignon technique was used for the semen freezing. After descongelation at $37^{\circ} \mathrm{C}$ during 30 seconds, the content of each slat was diluted in Beltsville Thawing Solution (BTS). They were analysed in optical microscopy, the vigour and the spermatic motility, after descongelation (M1), dilution (M2) , 10 minutes (M3) and 2 hours of incubation (M4) at $37^{\circ} \mathrm{C}$. The acrossome morphology was analyzed in M3. The Mann Whitney's test was used with the Lineal General Models in Statistical Analysis System Program (HEALTHY 6.03, 1988) at 5\% $(\mathrm{p}<0,05)$.
\end{abstract}

Keywords: boars, semen, freezing, spermatozoids, sensibility.

\section{Introdução}

As diferentes técnicas empregadas na congelação do sêmen do varrão esbarra ainda em barreiras de ordem prática, apresentando particularmente uma grande variabilidade nos resultados de fertilidade de fêmeas inseminadas com doses de sêmen descongeladas (Pursel e Johnson, 1971; Paquignon e Courot, 1975; Paquignon et al., 1977; Toniolli et al., 1997). Esta variabilidade pode estar relacionada com os diferentes métodos que visam uma melhor recuperação do sêmen após a descongelação (Paquignon et al., 1974; Almlid et al., 1989; Holfo e Almlid, 1992; Eriksson e Rodriguez-Martinez, 2000).
Na espécie suína, alguns indivíduos apresentam uma melhor qualidade espermática após a descongelação (Larsson e Einarsson, 1976; Watson, 1995; Cerolini, 2001; Ohata et al., 2001). Parkinson e Whitfield (1987) relataram que a má qualidade espermática individual após a descongelação pode ser melhorada de acordo com o método de congelação utilizado. As variações na resistência dos espermatozoides de diferentes varrões, aos processos de congelação/ descongelação, permitem diferentes taxas de fertilidade utilizando-se um mesmo método (Larsson e Einarsson, 1976; Paquignon et al., 1977; Almlid et al., 1989; Eriksson e RodriguezMartinez, 2000). Este trabalho teve por objetivo identificar variação de resistência nos ejaculados de diferentes varrões utilizando-se um mesmo método de congelação.

* Laboratório de Reprodução Suína e Tecnologia de Sêmen - FAVET / Universidade Estadual do Ceará, Av. Paranjana, 1700 - Campus do Itaperi, Fortaleza, CE. CEP: 60.740-000, e-mail: toniolli@roadnet.com.br 


\section{Material e métodos}

\section{Animais e coleta do sêmen}

Foram utilizados ejaculados de quatro animais adultos, dois híbridos comerciais (Dalland) e dois puros (Duroc e Landrace), submetidos a um mesmo manejo e regime de duas coletas semanais. Cada reprodutor foi coletado 10 vezes pela técnica da mão enluvada com manequim. 0 sêmen foi coletado em frascos plásticos com capacidade para $500 \mathrm{~mL}$, previamente aquecidos a $37^{\circ} \mathrm{C}$ e protegidos com copo térmico, sendo utilizado o ejaculado total após separação da fração gelatinosa através de filtração em gaze especial. Após a coleta, o ejaculado foi analisado quanto à sua concentração espermática $\left(\times 10^{6} \mathrm{sptz} / \mathrm{mL}\right)$, volume $(\mathrm{mL})$, total de espermatozoides $\left(\times 10^{9}\right)$, vigor espermático (escore de 0 a 5 - Toniolli, 1996) e motilidade espermática (percentual de células móveis). Todos os ejaculados utilizados no experimento apresentaram valores acima de 3,5 e $80 \%$ para vigor e motilidade, respectivamente. Todos os animais coletados eram submetidos periodicamente a exames morfológicos encontrando-se dentro do padrão exigido pelo Colégio Brasileiro de Reprodução Animal (1998).

\section{Congelação e descongelação do sêmen}

O sêmen foi congelado pelo método de Paquignon et al. (1974). De cada ejaculado, foi retirado um total de $4 \times 10^{9} \mathrm{sptz}$, colocado em tubos de fundo cônico (Corning de $50 \mathrm{~mL}$ ) incubado em banho-maria a $30^{\circ} \mathrm{C}$ por 30 minutos. Em seguida, o sêmen in natura foi centrifugado a $800 \mathrm{~g}$ por 15 minutos para retirada do plasma seminal e adição do diluente de congelação. O sêmen diluído foi acondicionado em palhetas de $0,5 \mathrm{~mL}$, devidamente identificadas e vedadas com esferas plásticas. Visando a descongelação, as palhetas foram colocadas em descongelador automático a $37^{\circ} \mathrm{C}$ por 30 segundos.

\section{Momentos de análise após descongelação do sêmen}

O sêmen foi analisado em 4 momentos diferentes:

1) Logo após a descongelação, analisando-se uma gota do conteúdo da palheta entre lâmina e lamínula (M1);

2) $\mathrm{O}$ restante do conteúdo da palheta foi diluído em $1,0 \mathrm{~mL}$ no Betsville Thawing Solution (BTS), a $37^{\circ} \mathrm{C}$ sendo avaliada em seguida (M2);

3) A terceira análise foi feita após 10 minutos de incubação do sêmen diluído no BTS (M3);

4) $O$ quarto momento de análise foi após 120 minutos de incubação no diluído no BTS (M4).

\section{Características analisadas}

Vigor e motilidade espermática: as características de vigor (VIG) e motilidade (MOT) foram avaliadas através de leituras feitas à luz da microscopia óptica após 10 minutos de incubação do sêmen a $37^{\circ} \mathrm{C}$, que se estendeu até 120 minutos para aplicação do teste de termorresistência (TTR) através da análise do vigor espermático, subtraindo-se 0 resultado da primeira leitura pelo da segunda, dividindo-se pelo primeiro resultado e multiplicando-se por 100. Valor final expresso em percentagem. Estas duas características (VIG e MOT) foram avaliadas em todos os momentos (M1, M2, M3 e M4).

Morfologia: Uma amostra de sêmen $(0,1 \mathrm{~mL})$ foi retirada para análise apenas após 10 minutos de incubação a $37^{\circ} \mathrm{C}$ (M3), diluída em partes iguais em solução salina formolizada (0,9\% de $\mathrm{NaCl}$ e 0,4\% de formol), para avaliação do percentual de células com acrossomo intacto. As análises foram realizadas colocando-se $15 \mu \mathrm{L}$ da solução entre lâmina e lamínula, onde foram contadas 200 células por lâmina em microscópio óptico com contraste de fase (1000x). Os espermatozoides foram classificados em 5 diferentes categorias, segundo Pursel et al., 1972a). Com acrossomo normal (NAR): contornos nítidos, regulares, superfície lisa e de forma arredondada; b) Com perda de acrossomo (PAR): superfície fracamente visualizada, vesiculada, transparente, com o acrossomo em degeneração; c) Com acrossomo edemaciado (EAR): com um inchaço na região acrossômica; d) Com acrossomo danificado (DAR): superfície acrossômica bem visível porém irregular, com invaginações ou evaginações; e) Sem acrossomo (SAR): acrossomo não visualizado e região equatorial bem definida.

\section{Análise estatística}

O delineamento experimental utilizado foi o de blocos ao acaso com a análise estatística feita pela avaliação das médias e desvios-padrões, com aplicação de testes de análise de variância. O teste proposto foi o de Mann Whitney para a comparação entre grupos. A análise das diferenças entre médias foi feita por variância multifatorial usando-se o General Linear Models do programa Statistical Analysis System (SAS 6.03, 1988), com um intervalo de confiança de $5 \%(p<0,05)$.

\section{Resultados e discussão}

\section{Vigor e motilidade espermática pós-descongelação}

Para a característica vigor espermático, em todos os momentos de análise, o animal 2 apresentou valor inferior $(p<0,05)$ em relação aos outros reprodutores (Figura 1). Os maiores valores de VIG foram encontrados no sêmen dos animais 3 e $4(p<0,05)$. Entretanto, após o TTR (M4), o animal 4 apresentou o maior valor de VIG em relação a todos os outros (Figura 1, p<0,05). Por outro lado, o animal 1 foi melhor em todas as etapas pós-descongelação apenas em relação ao animal 2. Os animais 3 e 4 apresentaram os mesmos valores médios de VIG durante o M1, (animal 3 e 4 =3,3) e M2 (animal 3 e 4 = 2,6, fig. 1). Após dez minutos de incubação diluído no BTS (M3), o animal $3(3,3)$ e o $4(3,4)$, não apresentaram diferenças ( $p>0,05)$ entre si. Entretanto, no M4, o animal 4 apresentou maior valor de VIG $(2,8)$ em relação aos outros reprodutores (Figura 1, $\mathrm{p}<0,05$ ).

As diferenças individuais na sensibilidade dos espermatozoides de diferentes machos podem estar relacionadas com o choque osmótico que as células sofrem durante a formação de cristais de gelo, aumento da pressão osmótica e alteração da permeabilidade da membrana (Watson, 2000), influenciando desta forma na sobrevivência e a capacidade 


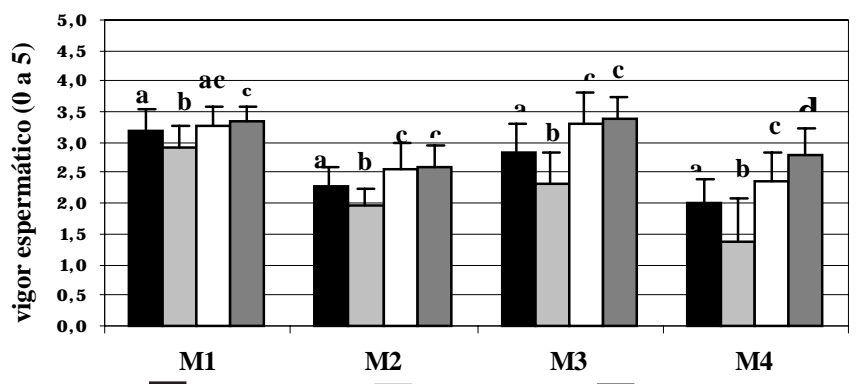

Animal $1=\square \quad$ Animal $2=\square \quad$ Animal $3=\square \quad$ Animal $4=\square$

Letras diferentes indicam diferenças entre os animais, dentro de cada momento $(p<0,05)$.

Figura 1: Vigor espermático individual do ejaculado de diferentes varrões, em quatro momentos de avaliação (M1, M2, M3, M4) após a descongelação do sêmen.

de fertilização dos espermatozoides (Holt, 2000). Portanto, os diferentes resultados do vigor espermático entre os reprodutores neste trabalho podem ter sido devido à resistência individual da membrana plasmática do espermatozoide de cada um dos varrões estudados.

Os resultados de VIG encontrados nos ejaculados do animal 2 , evidenciaram uma baixa resistência à congelação. Sabese que a magnitude na perda da qualidade do sêmen de varrões após a criopreservação, pode estar diretamente relacionada com a baixa qualidade inicial dos ejaculados in natura (Cerolini et al., 2001). Portanto, as diferenças encontradas na congelabilidade individual entre os animais deste trabalho não podem estar relacionadas com as características dos ejaculados in natura, devido a uma triagem inicial do sêmen conforme descrito na metodologia, e sim a fatores intrínsecos relativos às características individuais dos espermatozoides de diferentes reprodutores.

Para a característica motilidade espermática, o reprodutor 2 apresentou o menor valor $(61,1 \% ; p<0,05)$ no $\mathbf{M} 1$ em relação aos outros animais e permaneceu assim durante todos os outros momentos de avaliação. Para os reprodutores 1,3 e 4 , logo após a descongelação (M1), não houve diferenças entre si (animal $1=66,6 \%$, animal $3=69 \%$ e animal $4=69,2 \%$; Figura 2). Entretanto, em M2 e M3 os animais 3 e 4 apresentaram valores de MOT maiores do que os animais 1 e $2(p<0,05)$ não havendo entretanto, diferença significativa entre eles (animal $3=49,5 \%$ e animal $4=49,2 \%$, no $\mathbf{M} 2$, Figura 2).

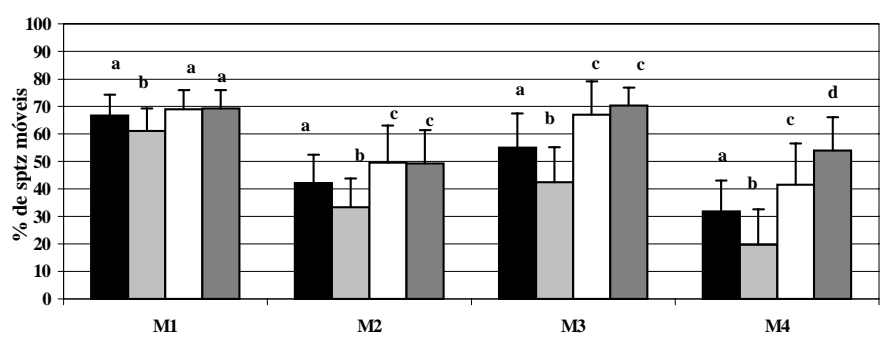

Animal $1=\square \quad$ Animal $2=\square \quad$ Animal $3=\square \quad$ Animal $4=\mathbf{Q}$ Letras diferentes indicam diferenças entre os animais, dentro de cada momento $(\mathrm{p}<0,05)$.

Figura 2: Motilidade espermática individual do ejaculado de diferentes varrões, em quatro momentos de avaliação (M1, M2, M3, M4) após a descongelação do sêmen.
Características como a frequência de ejaculação e o tempo de permanência dos espermatozoides no epidídimo, estão relacionados com a variabilidade na resposta individual ao processo de criopreservação (Watson, 1995). Uma vez que todos os reprodutores testados neste trabalho foram submetidos a uma mesma frequência de coleta (duas vezes por semana), diferenças encontradas como os resultados obtidos com o sêmen do animal 2, podem também estar relacionadas com características individuais de sensibilidade espermática e técnica de congelação utilizada. Esta possível correlação deve ser verificada a posteriori.

Todos os animais apresentaram diferenças individuais entre a qualidade dos espermatozoides no M4, sendo que o animal 4 foi aquele que melhor suportou o período de incubação do sêmen pós-descongelação, apresentando maior número de células móveis em relação a todos os outros (animal $1=$ $31,9 \%$, animal $2=19,7 \%$, animal $3,=41,5 \%$, animal $4=$ $54 \% ; p<0,05)$. O animal 2 apresentou o menor valor de MOT $(p<0,05)$, evidenciando uma menor resistência espermática ao processo de congelação em relação aos outros reprodutores. Este resultado pode estar relacionado com mudanças nos lipídeos de membrana durante o trânsito epididimário (Watson, 1981), onde os espermatozoides adquirem resistência ao choque térmico, fato este que pode influenciar na diferença individual dos ejaculados ao processo de criopreservação.

Baixas concentrações espermáticas no acondicionamento do ejaculado podem aumentar a sensibilidade das células ao choque térmico. Os melhores resultados de espermatozoides móveis após a descongelação são obtidos com concentrações finais variando entre 400 e $800 \times 10^{6} / \mathrm{mL}$ (Paquignon e Courot, 1975). Entretanto, neste trabalho foram utilizados $200 \times 10^{6} \mathrm{sptz} / \mathrm{mL}$ sendo os resultados de MOT encontrados no animal 4 (54\% em M4, figura 2), melhores do que os obtidos por estes autores (20\%). Mais uma vez as diferenças pós-descongelação encontradas neste trabalho não parecem estar relacionadas apenas com o processo de criopreservação, bem como também a fatores individuais, fato este encontrado também confirmado por outros autores (Watson, 1995; Cerolini et al., 2000).

Os diferentes resultados entre os varrões, podem estar relacionados com a resistência individual dos ejaculados ao estresse osmótico sofrido pelos espermatozoides durante o processo de congelação, descongelação e após a diluição com BTS. Logo após a descongelação, as células espermáticas saem de um diluente hiperosmótico (gema de ovo/glicose), para um isosmótico (BTS) (Paquignon et al., 1986), onde o animal 2 apresentou os menores valores de VIG e MOT, podendo indicar uma produção de células com menor resistência osmótica. Caiza de la Cueva et al. (1997) relataram um efeito individual significativo na viabilidade dos espermatozoides após serem submetidos a uma brusca mudança de um meio hiperosmótico para um meio isosmótico indicando que o sêmen de diferentes indivíduos pode interferir na resposta do espermatozoide suíno ao estresse hiperosmótico, fato este constatado nos resultados deste trabalho.

Após as duas horas de incubação, o varrão 2 apresentou menores valores de VIG e MOT, indicando uma baixa taxa de sobrevivência dos espermatozoides deste animal em relação aos outros. Larsson e Einarsson (1976) reportaram diferen- 
tes resultados de motilidade espermática entre varrões, com um maior decréscimo no percentual de células móveis após a incubação estar relacionado com resultados mais baixos de fertilidade. Outros autores verificaram que, espermatozoides com curta sobrevivência in vitro também possuem curta duração dentro do trato genital de fêmeas inseminadas com sêmen descongelado (Einarsson et al, 1973, Einarsson e Viring; 1973 Larsson et al., 1976), desta forma pode ser esperado que o varrão 4, por ter apresentado melhores resultados de VIG e MOT após descongelação pode apresentar espermatozoides com maior resistência ao choque térmico e provavelmente alcançar melhores resultados de fertilidade do que o animal 2.

\section{Morfologia espermática pós-descongelação}

Os resultados de morfologia do acrossoma estão apresentados na tabela 1. Houve uma recuperação de 45 a $50 \%$ das células com o acrossoma normal após a descongelação, em todos os animais testados. No entanto, o animal 2, ao contrário dos parâmetros de VIG e MOT, apresentou maior porcentagem de espermatozóides com NAR do que os animais 3 e 4 mas não foi diferente do animal 1 ( $p>0,05)$. Os animais 1 e 3 também não apresentaram diferenças entre si nos valores de NAR.

Tabela 1: Aspectos morfológicos individuais do acrossoma do sêmen diferentes varrões após descongelação, ressuspensão e incubação a $37^{\circ} \mathrm{C}$ durante 10 minutos

\begin{tabular}{lccccc}
\hline \multicolumn{7}{c}{ Defeitos do acrossoma (\%) } \\
\hline NAR & PAR & EAR & SAR & DAR \\
\hline (1) Landrace & $50,7^{\mathrm{ab}}$ & $39,1^{\text {ab }}$ & $7,2^{\mathrm{a}}$ & $2,5^{\mathrm{a}}$ & $0,5^{\mathrm{a}}$ \\
(2) Duroc & $52,3^{\mathrm{b}}$ & $36,7^{\mathrm{b}}$ & $6,5^{\mathrm{a}}$ & $3,3^{\mathrm{a}}$ & $1,2^{\mathrm{a}}$ \\
(3) Dalland 69 & $44,9^{\mathrm{a}}$ & $42,0^{\mathrm{a}}$ & $9,4^{\mathrm{b}}$ & $4,3^{\mathrm{a}}$ & $0,5^{\mathrm{a}}$ \\
(4) Dalland 70 & $45,9^{\mathrm{a}}$ & $40,3^{\mathrm{a}}$ & $8,4^{\mathrm{b}}$ & $4,4^{\mathrm{a}}$ & $1,0^{\mathrm{a}}$ \\
\hline
\end{tabular}

Obs.: Letras sobrescritas diferentes indicam diferenças estatísticas dentro das colunas $(p<0,05)$.

Foi observado um efeito individual com relação aos resultados de morfologia do acrossoma, entretanto, os animais 1 e 2 foram os que apresentaram maior número de NAR e menor número de PAR. No entanto, estes animais apresentaram menores valores de VIG e MOT em relação aos animais 3 e 4 . Estas observações na morfologia espermática podem indicar que fatores inerentes aos próprios indivíduos são mais decisivos para os resultados após a descongelação do que qualquer outro intrínseco à técnica de criopreservação (Paquignon e Courot, 1975; Almlid et al., 1989; Eriksson e Rodriguez-Martinez, 2000).

Apesar dos animais 1 e 2 terem apresentado maiores percentuais de células com acrossoma intacto, a técnica utilizada para verificação das características morfológicas neste trabalho, não permitiu distinguir a viabilidade espermática.
Logo, não se pode afirmar apenas com este parâmetro que estes animais possuem maior resistência ao choque térmico, pois as células analisadas que estavam com NAR poderiam não estar viáveis no momento (M3) em que foram retiradas e fixadas para esta análise.

Os resultados de MOT e NAR encontrados no varrão 2 corroboram com os de Eriksson e Rodriguez-Martinez (2000), que observaram maior número de células com a membrana acrossomal íntegra do que de células móveis. Tais resultados indicam a necessidade da utilização de diferentes parâmetros para verificar a resistência dos espermatozoides pós-descongelação. No M3 o varrão 2 apresentou número significativamente mais baixo de células móveis (animal $2=$ $42 \%$ e animal $4=70 \% ; p<0,05)$ e maior número de células com acrossoma intacto (52\%). Logo, pode-se estimar que $42 \%$ de seus espermatozoides estavam móveis; entretanto, apenas $52 \%$ das células apresentavam com acrossoma intacto enquanto o animal 4 apresentou $70 \%$ de suas células móveis (M3) com $45 \%$ das suas células com NAR. Portanto, pode-se estimar que 0 animal 4 apresentou melhor qualidade espermática após a descongelação.

O animal 2 apresentou uma percentagem menor de espermatozoides com PAR do que os animais 3 e $4(p<0,05)$. Entretanto, não foi diferente do animal 1 . Não houve diferenças individuais entre os animais 1 , 3 e 4 no número de espermatozoides que apresentaram perda de acrossoma (PAR). A característica PAR foi a alteração morfológica mais observada após a descongelação, onde 36 a $40 \%$ dos espermatozoides de todos os animais apresentaram o acrossoma em processo de perda (PAR).

Com relação ao edema de acrossoma (EAR), os animais $1 \mathrm{e}$ 2 apresentaram um número menor $(p<0,05)$ de células com esse defeito (animal $1=7,2 \%$ e animal $2=6,5 \%$ ) em relação aos animais 3 e 4 (animal $3=9,4 \%$ e animal $4=8,4 \%$ ). Em todos os reprodutores foi observada uma pequena proporção de células sem acrossoma (SAR $=2,5$ a 4,4\%, Tabela 1), não havendo diferenças entre os animais testados $(p>0,05)$. No que diz respeito ao acrossoma danificado (DAR), este foi o defeito que apareceu com menor proporção nos espermatozoides de todos os animais estudados, com $1 \%$ ou menos, nos animais 1,3 e 4 e 1,2\% no animal 2. Com relação a esta característica, também não foram observadas diferenças entre os animais em estudo $(p>0,05)$.

\section{Conclusões}

O uso do sêmen suíno criopreservado em programas de inseminação artificial com a obtenção de bons resultados de fertilidade, deve estar obrigatoriamente relacionado com o fator individual nos resultados pós-descongelação. Desta forma, a identificação e seleção de animais com ejaculados mais resistentes ao processo de congelação é necessária. O efeito individual dos ejaculados na criopreservação do sêmen de suínos, afeta a qualidade dos espermatozoides após a descongelação, tornando-se necessário uma análise prévia da congelabilidade dos ejaculados dos diferentes reprodutores a serem selecionados para o processo. 


\section{Referências}

ALMLID, T.; CLARKE, R. N.; PURSEL, V. G.; JOHSON, L. A. EffectivinesS of in vitro methods for predicting in vivo fertilizing capacity of boar spermatozoa cryopreserved with $2 \%$ or $4 \%$ glycerol. Zuchthyg., v. 24, p. 8-15. 1989.

CAIZA DE LA CUEVA F., RIGAU, T., PUJOL R., PIEDRAFITA, J., RODRÍGUEZ-GIL, J. E. Resistance to hyperosmotic stress in boar spermatozoa: the role of the ionic pumps and the relationship with cryosurvival. Anim. Reprod. Sci., v. 48, p. 301-315, 1997.

CEROLINI, S., MALDJIAN, A., PIZZI, F., GLIOZZI T. M. Changes in sperm quality and lipid composition during cryopresrvation of boar semen. J. Reprod. Fertil., v. 121, p. 395-401, 2001.

CEROLINI, S., MALDJIAN, A., SURAI, P., NOBLE, R. Viability, susceptibility to peroxidation and fatty acid composition of boar semen during liquid storage. Anim. Reprod. Sci., v. 58, p. 99-111, 2000.

CBRA. Manual para exame andrológico e avaliação de sêmen animal. 2. ed., Belo Horizonte: Colégio Brasileiro de Reprodução Animal, 1998. $49 \mathrm{p}$.

EINARSSON, S., VIRING, S. Distribution of frozen thawed spermatozoa in the reproductive tract of gilts at different time intervals after insemination. J. Reprod. Fertil., v. 32, p.117-120, 1973.

EINARSSON, S., SOOSALU, O., SWENSSON, T., VIRING, S. On the fertility and survival of deep frozen spermatozoa thawed in skim milk Acta Vet. Scand., v.13, p. 446-448, 1972.

ERIKSSON, B. M., RODRIGUEZ-MARTINEZ, H. Effect of freezing and twawing rates on the post-thaw viability of boar spermatozoa frozen in Flatpacks and Maxi-straws. Anim. Reprod. Sci., v. 63, p. 205-220, 2000.

HOFMO, P.O.; ALMLID, T. Recent developments in freezing of boar semen with special emphasis on cryoprotectants. Rep. Dom. An., supp.1, v. 26 p.111-122, 1992.

HOLT, W. V. Basic aspects of frozen storage of semen. Anim. Reprod. Sci., v. 62, p. 3-22, 2000.

LARSSON, K., EINARSSON, S. Influence of boars on the relationship between fertility and post-thawing sperm quality of deep frozen boar spermatozoa. Acta Vet. Scand., v. 17, p. 74-82, 1976.

LARSSON, K., EINARSSON, S., NICANDER, L. Influence of thawing diluent on vitality, acrosome morphology ultrastructure and ezyme release of deep frozen boar spermatozoa. Acta Vet. Scand., v. 17 p. 83-100, 1976.

OHATA, P. M., WENTZ, I., BERNARDI, M. L., CASTAGNA, C., BORTOLOZZO, F. P. Influência do período de equilíbrio précongelamento e plasma seminal na congelabilidade do sêmen suíno. CONGRESSO BRASILEIRO DE VETERINÁRIOS ESPECIALISTASEM SUÍNOS, 10., Proceedings... 2001, v. 2, p. 255-256, 2001.
PAQUIGNON, M.; MERGOUNIS, D.; COUROT, M.; DU MESNIL DU BUISSON, F. Technologie de la congélation de la semence de verrat: étude in vitro. J. Rech. Porc. France, v. 6, p. 71-76, 1974.

PAQUIGNON, M; COUROT, M. Survie des spermatozoides de verrat aprés décongélation. Effet du rythme de collectes, de la concentration et du taux de glycérol. Biol. An. Bioch. Bioph., v. 15, n. 3, p. 517-523, 1975.

PAQUIGNON, M., BUSSIERE, J., BARITEAU, F., COUROT, M. Resultats pratiques d'utilisation de semence congelée de verrat. J.Rech. Porc.France, v. 9, p. 27-34, 1977.

PAQUIGNON, M., QUELLIER, P., DACHEUX, J. L. Congélation du sperme de verrat: comparaison de differents diluers, techiques de préparation de la semence, mode de conditionnement et températures de décongélation. Ann. Zoot., v. 35, p.173-184, 1986.

PARKINSON, T. J., WHITFIELD, C. H. Optimisation of freezing conditions for bovine spermatozoa. Theriog., v. 27 p. 781-797, 1987.

PURSEL V.G; JOHNSON L.A. Fertility with frozen boar spermatozoa. J. An. Sci., v. 33, p. 265, 1971 (abstract).

PURSEL V.G.; JOHNSON L.A; RAMPACEK G.B. Acrosome morphology of boar spermatozoa incubated before cold shock. J. An. Sci., v. 34, p. 278-283, 1972

TARDIF, S., LAFOREST, J. P., CORNIER, N., BAILEY, J.L. The importance of porcine sperm parameters on fertility in vivo. Theriog., v. 52, p. 447-459. 1999

TONIOLLI, R. Pouvoir fécondant dês spermatozoïdes de verrat : amèlioration des conditions de conservation. Tours-France, 1996. 91 p. Tese de Doutorado, Nouzilly, France, 1996.

TONIOLLI, R., BUSSIERE, J., COUROT, M., COMBARNOUS, Y. Effect of indole-3 acetic acid (plant auxin) on boar sperm motility and pregnancy and prolificacy rates freezing and thawing. Reprod. Dom. An., v. 33, p. 33-38, 1998.

WATSON, P. F. Effects of cold shock on sperm cell membranes. In: Bwanga C. O. Cryopreservation of boar semen I: A literature review. Acta Vet. Scand., v. 32 p. 431-453, 1981.

WATSON, P.F. Recent developments and concepts in cryopreservation of spermatozoa and the assessment of their post-thawing function. Reprod. Fertil. Develop., p. 871-891, 1995.

WATSON, P. F. The causes of reduced fertility with cryopreserved semen. Ani. Reprod. Sci., v. 60-61, p. 481-492, 2000. 